\title{
A New Straightforward Method for Evaluating Singular Integrals
}

\author{
Md. Habibur Rahaman ${ }^{1,2}$, Md. Ashraful Huq ${ }^{1}$, M. Kamrul Hasan ${ }^{1}$ \\ ${ }^{1}$ Department of Mathematics, Rajshahi University of Engineering and Technology, Kazla, Rajshahi-6204, Bangladesh \\ ${ }^{2}$ Adarsho Karigori \& Banijjik College, Hatemkhan, Rajshahi-6000, Bangladesh
}

Email address:

habiburhpr@gmail.com (Md. H. Rahaman), uas.mukul@gmail.com (Md. A. Huq), mkh2502@yahoo.com (M. K. Hasan)

\section{To cite this article:}

Md. Habibur Rahaman, Md. Ashraful Huq, M. Kamrul Hasan. A New Straightforward Method for Evaluating Singular Integrals. Applied and Computational Mathematics. Vol. 4, No. 6, 2015, pp. 420-423. doi: 10.11648/j.acm.20150406.14

\begin{abstract}
A new more accurate straightforward method is presented for evaluating the singular integrals. A few methods in numerical analysis is useful for evaluating the integral where singularities arises, most of them uses extrapolation technique at singular point. This new method uses directly and gives better results and the Romberg integration of this formula converses faster than others previous methods.
\end{abstract}

Keywords: Numerical Integration, Singular Integrals, Lagrange’s Interpolation Formula, Romberg Integration

\section{Introduction}

Newton-Cotes formulas, such as Trapezoidal rule, Simpson's rules and Weddle's rule etc. cannot be use directly for integrals where the integrands become infinite at the ends of the intervals. However Gauss quadrature rules may used to evaluate such singular integrals. But it is a laborious task. Earlier Fox [1] used classical formulae for evaluating such integrals where the functional values at the singular points are extrapolated. Recently, Huq et al [2] developed a simple and straightforward method for evaluating singular integrals of the form

$$
\mathrm{I}=\int_{a}^{b} y(x) d x
$$

where $y(x)$ is singular at $x=a$ or $x=b$.

The aim of this article is to obtain a new straightforward formula for evaluating singular integrals and also obtain better result than other existing solutions.

\section{Derivation of the Formula}

Generally numerical integration formulae are reformed by utilizing an interpolation formula. The Trapezoidal rule, Simpson's rules etc. are established by Newton's forward formula. Recently Huq et al [2] has been used Lagrange's formula to derive an integration formula, e.g,

$$
\int_{x 0}^{x 3} y(x) d x=\frac{3 h}{4}\left[3 y_{1}+y_{3}\right], \text { where } h=\frac{x_{3}-x_{0}}{3}
$$

Considering three points $\mathrm{x}_{0}, \mathrm{x}_{1}, \mathrm{x}_{3}$ together with $\mathrm{x}_{1}=\mathrm{x}_{0}+\mathrm{h}$ and $x_{3}=x_{0}+3 h$. It is clear that formula (2) excludes $y_{0}$ and thus it is used directly when $\mathrm{y}(\mathrm{x})$ is singular at $\mathrm{x}_{0}$.

A general form of formula (2) in the interval $[a, b]$ is

$$
\int_{a}^{b} y(x) d x=\frac{3 h}{4}\left[3\left(y_{1}+y_{4}+y_{7}+\ldots\right)\right.
$$

where, $h=(b-a) / 3 n, n=1,2,3, \ldots$ In order to derive a more accurate formula using Lagrange's interpolation formula, we search various points randomly. We find several formulae of 4 nodes and to find optimum of them we observed that, the formula is suitable which coefficient of error term is minimum. In this circumstances we consider five unequal points $\mathrm{x}_{0}, \mathrm{x}_{1}, \mathrm{x}_{2}, \mathrm{x}_{3}$ and $\mathrm{x}_{4}$ together with $\mathrm{x}_{0}=0$, $\mathrm{x}_{1}=\mathrm{x}_{0}+\mathrm{h}, \mathrm{x}_{2}=\mathrm{x}_{0}+5 \mathrm{~h}, \mathrm{x}_{3}=\mathrm{x}_{0}+11 \mathrm{~h}, \mathrm{x}_{4}=\mathrm{x}_{0}+15 \mathrm{~h}$ in the interval $[a, b]$ and the formula has been taken the form

$$
\int_{x 0}^{x 4} y(x) d x=\frac{15}{448} \mathrm{~h}\left(75 \mathrm{y}_{1}+161 \mathrm{y}_{2}+175 \mathrm{y}_{3}+37 \mathrm{y}_{4}\right)
$$

Where $h=\frac{b-a}{15}$

The formula (4) is useful directly when $y(x)$ is nonsingular or lower singular.

On the contrary, another formula

$\int_{x_{0}}^{x_{4}} y(x) d x=\frac{15}{448} h\left(37 y_{0}+175 y_{1}+161 y_{2}+75 y_{3}\right)$ 
has been obtained by considering five points $\mathrm{x}_{0}, \mathrm{x}_{1}, \mathrm{x}_{2}, \mathrm{x}_{3}$ and $\mathrm{x}_{4}$ together with $\mathrm{x}_{0}=0, \mathrm{x}_{1}=\mathrm{x}_{0}+4 \mathrm{~h}, \mathrm{x}_{2}=\mathrm{x}_{0}+10 \mathrm{~h}, \mathrm{x}_{3}=\mathrm{x}_{0}+14 \mathrm{~h}$, $\mathrm{x}_{4}=\mathrm{x}_{0}+15 \mathrm{~h}$. Herein $\mathrm{x}_{4}$ has been ignored.

Clearly formula (5) is useful directly when $\mathrm{y}(\mathrm{x})$ is upper singular.

\section{Error of the Present Formula}

The error of formula (4) is calculated as

$$
-\frac{375 \mathrm{~h} 6 \mathrm{~F}^{(5)}[\xi]}{16}-\frac{1625 \mathrm{~h}^{7} \mathrm{~F}^{(6)}[\xi]}{7}-
$$

Hence the error of formula (4) is

$$
-\frac{375 \mathrm{~h} 6 \mathrm{~F}^{(5)}[\xi]}{16}
$$

\section{Examples}

\subsection{Consider a Singular Integral}

$$
\int_{0}^{1} \frac{1}{\sqrt{x}} d x
$$

In the case of the singular integral $\int_{0}^{1} \frac{1}{\sqrt{x}} d x$, here 0 is the singular point

Using the formula (4) we obtain the approximate value of the integral (8) is $1.80958,1.86535$ and 1.90479 for $h=\frac{1}{15}$, $h=\frac{1}{30}$ and $h=\frac{1}{60}$. The exact value of this integral is 2 . Earlier Fox [1] measured 1.577350,1.698844 and 1.786461 for $h=\frac{1}{4}, h=\frac{1}{8}$ and $h=\frac{1}{16}$ and using extrapolation technique at $x=0$. Recently deriving a straightforward method Huq [2] measured 1.67961468, 1.77322680 and 1.83961679 for $h=\frac{1}{6}, h=\frac{1}{12}$ and $h=\frac{1}{24}$ for the same integral (8).

Both Fox [1] and Huq [2] presented a Romberg integration scheme of these results has been given in Table 4.1(a) and Table 4.1(b). Then the new results and its Romberg integration scheme have been given in Table

\begin{tabular}{|c|c|c|}
\hline$U\left(\frac{1}{4}\right)=1.577350$ & $U\left(\frac{1}{4}, \frac{1}{8}\right)=1.992156$ & \\
\hline$U\left(\frac{1}{8}\right)=1.698844$ & & $U\left(\frac{1}{4}, \frac{1}{8}, \frac{1}{16}\right)=1.999931$ \\
\hline$U\left(\frac{1}{16}\right)=1.786461$ & $U\left(\frac{1}{8}, \frac{1}{16}\right)=1.997987$ & \\
\hline
\end{tabular}
4.1(c).

Table 4.1(a). Numerical values of the integral 4.1 presented by Fox [1].

Table 4.1(b). Numerical values of the integral 4.1 presented by Huq [2].

\begin{tabular}{l|ll}
\hline$H\left(\frac{1}{6}\right)=1.67961468$ & $H\left(\frac{1}{6}, \frac{1}{12}\right)=1.99922645$ & \\
$H\left(\frac{1}{12}\right)=1.77322680$ & $H\left(\frac{1}{12}, \frac{1}{24}\right)=1.99989640$ & $H\left(\frac{1}{6}, \frac{1}{12}, \frac{1}{24}\right)=1.99994107$ \\
$H\left(\frac{1}{24}\right)=1.83961679$ & \\
\hline
\end{tabular}

Table 4.1(c). Numerical values of the integral 4.1 presented by new formula.

\begin{tabular}{lll}
\hline$N\left(\frac{1}{15}\right)=1.80958$ & $N\left(\frac{1}{15}, \frac{1}{30}\right)=1.99999013135$ & $N\left(\frac{1}{15}, \frac{1}{30}, \frac{1}{60}\right)=1.999999726$ \\
$N\left(\frac{1}{30}\right)=1.86535$ & $N\left(\frac{1}{30}, \frac{1}{60}\right)=1.99999942609$ & \\
$N\left(\frac{1}{60}\right)=1.90479$ & & \\
\hline
\end{tabular}

\subsection{A Singular Integral}

$$
\int_{0}^{1} x \ln x d x
$$

Using the formula (4) we obtain the approximate value of the integral (9) is 0.250675 . The exact value of this integral is 0.25 .

Both Fox [1] and Huq [2] presented a Romberg integration scheme of these results has been given in Table 4.2(a) and Table 4.2(b). Then Romberg integration scheme of the new result has been given in Table 4.2(c).

Table 4.2(a). Numerical values of the integral 4.1 presented by Fox [1].

\begin{tabular}{lll}
\hline$U\left(\frac{1}{4}\right)=0.281168$ & $U\left(\frac{1}{4}, \frac{1}{8}\right)=0.246632$ & \\
$U\left(\frac{1}{8}\right)=0.2595832$ & $U\left(\frac{1}{8}, \frac{1}{16}\right)=0.249478$ & \\
$U\left(\frac{1}{16}\right)=0.2528464$ & $\left.\frac{1}{16}\right)=0.250005$ \\
\hline
\end{tabular}


Table 4.2(b). Numerical values of the integral 4.1 presented by Huq [2].

\begin{tabular}{l|ll}
\hline$H\left(\frac{1}{6}\right)=0.25667294$ & $H\left(\frac{1}{6}, \frac{1}{12}\right)=0.24877579$ & \\
$H\left(\frac{1}{12}\right)=0.25173722$ & $H\left(\frac{1}{12}, \frac{1}{24}\right)=0.24966676$ & $\left.\frac{1}{12}, \frac{1}{24}\right)=0.25000649$ \\
$H\left(\frac{1}{24}\right)=0.25044318$ & & \\
\hline
\end{tabular}

Table 4.2(c). Numerical values of the integral 4.1 presented by new formula.

\begin{tabular}{lll}
\hline$N\left(\frac{1}{15}\right)=0.250675$ & $N\left(\frac{1}{15}, \frac{1}{30}\right)=0.250001$ & $N\left(\frac{1}{15}, \frac{1}{30}, \frac{1}{60}\right)=0.25000000922$ \\
$N\left(\frac{1}{30}\right)=0.250169$ & $N\left(\frac{1}{30}, \frac{1}{60}\right)=0.25000002661$ & \\
$N\left(\frac{1}{60}\right)=0.250042$ &
\end{tabular}

\subsection{A Integral without Singular Point}

$$
\int_{0}^{1} \sqrt{x} d x
$$

Choosing $h=\frac{1}{15}, h=\frac{1}{30}$ and $h=\frac{1}{60}$, formula (4) has value of the integral (10) is $0.667811,0.667072$ and 0.66681 . The exact value of this integral is $2 / 3$.

Both Fox [1] and Huq [2] presented a Romberg integration scheme of these results has been given in Table 4.3(a) and Table 4.3(b). Then Romberg integration scheme of the new result has been given in Table 4.3(c).

been utilized and measured respectively the approximate

Table 4.3(a). Numerical values of the integral 4.1 presented by Fox [1]

\begin{tabular}{ll|l}
$U\left(\frac{1}{4}\right)=0.6830125$ & $U\left(\frac{1}{4}, \frac{1}{8}\right)=0.667488$ & \\
$U\left(\frac{1}{8}\right)=0.672977$ & $U\left(\frac{1}{8}, \frac{1}{16}\right)=0.666875$ & \\
$U\left(\frac{1}{16}\right)=0.6690322$ & & \\
\hline
\end{tabular}

Table 4.3(b). Numerical values of the integral 4.1 presented by Huq [2].

\begin{tabular}{l|ll}
$H\left(\frac{1}{6}\right)=0.67266767$ & $H\left(\frac{1}{6}, \frac{1}{12}\right)=0.66671457$ & $H\left(\frac{1}{6}, \frac{1}{12}, \frac{1}{24}\right)=0.66665901$ \\
$H\left(\frac{1}{12}\right)=0.66881931$ & $H\left(\frac{1}{12}, \frac{1}{24}\right)=0.66667290$ & \\
$H\left(\frac{1}{24}\right)=0.66743177$ &
\end{tabular}

Table 4.3(c). Numerical values of the integral 4.1 presented by new formula.

$\begin{array}{lll}N\left(\frac{1}{15}\right)=0.667811 & N\left(\frac{1}{15}, \frac{1}{30}\right)=0.666667065663 & N\left(\frac{1}{15}, \frac{1}{30}, \frac{1}{60}\right)=0.666666667455 \\ N\left(\frac{1}{30}\right)=0.667072 & N\left(\frac{1}{30}, \frac{1}{60}\right)=0.666666686772 & \\ N\left(\frac{1}{60}\right)=0.66681 & \end{array}$

\subsection{A Integral without Singular Point}

$$
\int_{0}^{1} \sqrt{x(1-x)} d x
$$

Choosing $h=\frac{1}{15}, h=\frac{1}{30}$ and $h=\frac{1}{60}$ formula (4) has been utilized and measured respectively the approximate value of the integral (11) is 0.3839114432057704 ,
0.3896170243758529 and 0.39161400102331123 . The exact value of this integral is $\frac{\pi}{8}$.

Both Fox [1] and Huq [2] presented a Romberg integration scheme of these results has been given in Table 4.4(a) and Table 4.4(b). Then Romberg integration scheme of the new result has been given in Table 4.4(c).

Table 4.4(a). Numerical values of the integral 4.1 presented by Fox [1].

\begin{tabular}{ll|l}
\hline$U\left(\frac{1}{4}\right)=0.433012$ & $U\left(\frac{1}{4}, \frac{1}{8}\right)=0.393423$ & \\
$U\left(\frac{1}{8}\right)=0.407420$ & $U\left(\frac{1}{8}, \frac{1}{16}\right)=0.392834$ & $U\left(\frac{1}{4}, \frac{1}{8}, \frac{1}{16}\right)=0.392708$ \\
$U\left(\frac{1}{16}\right)=0.397991$ & & \\
\hline
\end{tabular}


Table 4.4(b). Numerical values of the integral 4.1 presented by Huq [2].

\begin{tabular}{lll}
\hline$H\left(\frac{1}{6}\right)=0.379031$ & $H\left(\frac{1}{6}, \frac{1}{12}\right)=0.392755$ & $H\left(\frac{1}{6}, \frac{1}{12}, \frac{1}{24}\right)=0.3927$ \\
$H\left(\frac{1}{12}\right)=0.387903$ & $H\left(\frac{1}{12}, \frac{1}{24}\right)=0.39271$ & \\
$H\left(\frac{1}{24}\right)=0.39101$ & & \\
\hline
\end{tabular}

Table 4.4(c). Numerical values of the integral 4.1 presented by new formula.

$\begin{array}{lll}N\left(\frac{1}{15}\right)=0.383911 & N\left(\frac{1}{15}, \frac{1}{30}\right)=0.392738 & N\left(\frac{1}{15}, \frac{1}{30}, \frac{1}{60}\right)=0.392699 \\ N\left(\frac{1}{30}\right)=0.389617 & N\left(\frac{1}{30}, \frac{1}{60}\right)=0.392706 & \\ N\left(\frac{1}{60}\right)=0.391614 & & \end{array}$

\section{Result and Discussions}

Fox [1] is not simple and straightforward for evaluating such type of singular integrals. Huq et al [2] is a simple and straightforward method which is better than the other existing methods for evaluating singular integrals.

Also from the above Tables $4.1-4.4$, it is clear that the Romberg integration scheme of the new method converges faster as well as gives more accurate result than Fox [1] and Huq [2] formula.

\section{References}

[1] L. Fox, Romberg integration for a class of singular integrand, Computer. Journal. 10(1) (1967), pp87-93.

[2] M. A. Huq, M. K. Hasan, M. M. Rahman and M. S. Alam, A Simple and Straightforward Method for evaluating some singular integrals, Far East Journal of Mathematical Education $\mathrm{V}-7, \mathrm{~N}-2,2011$, pp 93-103.

[3] E. A. Alshina, N. N. Kalitkin, I. A. Panin and I. P. Poshivaiol, Numerical integration of functions with singularities, Doki. Math. 74(2) (2006), pp 771-774.
[4] Numerical Mathematical Analysis, By James Blaine Scarborough, Publisher: Johns Hopkins Press, 1966ISBN0801805759, 9780801805752.

[5] Introductory Methods of Numerical Analysis, By S. S. Sastry, Prentice-Hall on India, New Delhi. ISBN: 8120327616, 9788120327610 .

[6] L. M. Delves, The numerical evaluation of principal value integrals, Comput. J. 10(4) (1968), pp 389-391.

[7] B. D. Hunter, The numerical evaluation of Cauchy principal values of integrals by Romberg integration, Numer. Math. 21(3) (1973) pp 185-192.

[8] H. W Stolle and R. Strauss, "On the Numerical Integration of Certain Singular Integrals”, Computing, Vol. 48(2), (1992), pp177-189.

[9] Linz, P. "On the approximate computation of certain strongly singular integrals." Computing35, 345-353 (1985).

[10] D. F. Paget, "The numerical evaluation of Hadamard finitepart integrals", Numerische Mathematik, Volume 36, Issue 4, pp 447-453, (1981). 